\title{
Traffic Rules Encoding Using Defeasible Deontic Logic
}

\author{
Hanif Bhuiyan ${ }^{\mathrm{a}, \mathrm{b}}$, Guido Governatori ${ }^{\mathrm{a}, 1}$, Andy Bond ${ }^{\mathrm{b}}$, Sebastien Demmel ${ }^{\mathrm{b}}$, \\ Mohammad Badiul Islam ${ }^{\mathrm{a}}$, Andry Rakotonirainy ${ }^{\mathrm{b}}$ \\ a Data61, CSIRO \\ ${ }^{\mathrm{b}}$ Queensland University of Technology (QUT), Centre for Accident Research and Road \\ Safety (CARRS-Q), Queensland, Australia
}

\begin{abstract}
Automatically assessing driving behaviour against traffic rules is a challenging task for improving the safety of Automated Vehicles (AVs). There are no AV specific traffic rules against which AV behaviour can be assessed. Moreover current traffic rules can be imprecisely expressed and are sometimes conflicting making it hard to validate AV driving behaviour. Therefore, in this paper, we propose a Defeasible Deontic Logic (DDL) based driving behaviour assessment methodology for AVs. DDL is used to effectively handle rule exceptions and resolve conflicts in rule norms. A data-driven experiment is conducted to prove the effectiveness of the proposed methodology.
\end{abstract}

Keywords. Automated Vehicle, Traffic Rules, Defeasible Deontic Logic, Assessment.

\section{Introduction}

Automated Vehicles (AVs) are one of the most remarkable and highly anticipated technological developments of this century. This technology where AVs are programmed to drive according to traffic rules [1] can be seen as a solution to improve road safety and prevent traffic violation [2]. Thus one of the challenges is how to assess AV behaviour with respect to traffic rules.

The main problem is that, currently, there is no separate and comprehensive regulatory framework for AVs [3]; thus there is no specific (traffic) regulation to specifically assess the AVs behaviour. Although researchers have speculated that the current regulatory framework may handle AVs in existing transport system situations, it remains unclear whether all existing traffic rules are (directly) applicable to AVs. Leens and Lucivero mentioned that the current traffic rule model might be incomplete for the AV for some driving scenarios [1]. For example, in the current Queensland traffic rules ${ }^{2}$, there are some vague expressions (e.g., "can safely overtake", "overtake when there is a clear view", etc.), which are almost impossible for an AV to follow [4] without additional parameters clarifying the meaning for the context and environment in which an AV is situated. Also, it may not be possible for AVs to properly follow rules which are related to conflicting situations [5] and exceptions.

1* Corresponding Author: Guido Governatori, Data61, CSIRO, Brisbane, Australia; Email:Guido.Governatori@data61.csiro.au

${ }^{2}$ https://www.legislation.qld.gov.au/view/html/inforce/current/sl-2009-0194 
Therefore there is the need to develop a methodology to assess the AV behaviour by bridging the gap between traffic rules and AV knowledge processing. In this paper, we propose such a methodology by first encoding traffic rules in a machine-computable (MC) format that can be used to address the above-mentioned issues to assess AV driving behaviour.

Traffic rules include thousands of provisions and complex norms. This makes the encoding task challenging. Therefore, in this research, we use Defeasible Deontic Logic (DDL) to encode traffic rules. DDL is the combination of defeasible logic and deontic logic. DDL has been successfully used in legal reasoning to handle norms and exceptions, and it does not suffer from problems affecting other logics used for reasoning about compliance and norms [6]. DDL is an effective logical approach to solve the conflicting situation in norms as it works based on defeasible logic using a suitable variant.

In this paper, the discussion on the methodology for assessing AV driving behaviour is based on Queensland overtaking traffic rules ${ }^{3}$. We choose overtaking traffic rules as it is one of the most challenging traffic rules which has several complicated conditions with multiple facets.

\section{Related Work}

In general, traffic rules are expressed in natural language and are created for human drivers. Traffic rules are often very detailed and complex and, therefore, it is a big challenge to encode them. Other research has addressed the challenges of traffic rule encoding for different purposes such as driving assistance systems [7], driving context modelling [8], traffic situation representation [9], etc. Some significant related research work about traffic rules encoding for assessing AV behaviour are given below.

In [4], Isabelle logic theorem is proposed to encode traffic rules to monitor the AV behaviour. This research aims to use monitoring to ensure that AV obeys traffic rules. To do that, traffic rules are codified into Linear Temporal Logic (LTL) using High Order Logic (HOL). A verified checker is used to check the compliance of the AV behaviour with the encoded traffic rules. To analyze the data, the recorded information is modelled as discrete-time runs.

In [10], an expert system to encode traffic rules for controlling the autonomous vehicle in certain situations is proposed. This expert system consists of data processing algorithms, multidimensional databases, and a cognitive model of traffic objects and their relationships. To encode traffic rules, data are grouped into two sets. One set consists of traffic lights, road markings, road signs, road types, etc. Another dataset consists of around 800 traffic rules.

In [11], an encoding method for traffic rules was proposed to keep the autonomous vehicle accountable. Three major steps consolidate this methodology. First, legal analysis alleviates the implicit redundancy from the legal text. Next, it explicitly sorts out the responsibility of the $\mathrm{AV}$ and the user and then breaks the rules into logical predicate precursors. One of the major aims of this work is to give the opportunity to further develop in the expressivity of rules (translated traffic rules) by using Higher Order Language (HOL).

In [12], a system, Mivar, is introduced that can monitor vehicle activities in real-time and can also inform the driver about the violations of traffic rules. The Mivar system

${ }^{3}$ https://www.legislation.qld.gov.au/view/html/inforce/current/sl-2009-0194\#pt.11-div.3 
consists of three main modules: trajectory control system (lane position, a safe distance from other vehicles, etc.), a simplified technical vision system (road situation in real-time), and a decision support system (DSS).

Although a few studies work on monitoring mechanisms on the AV activities to verify the AV behaviour against traffic rules $[12,4]$. However, none of them solve the issues of handling exceptions and resolving conflicting situation of traffic rules. However, these are important variant features and can create challenges while assessing the AV behaviour against traffic rules. In comparison to both of these works and other abovementioned works, we have proposed a DDL based methodology that can validate the AV behaviour against traffic rules more effectively by efficiently handling the rule exceptions and resolving conflicts in the traffic rules.

\section{Driving Behaviour Assessment}

The flow diagram of driving behaviour assessment methodology is shown in Figure 1. The proposed methodology consists of three modules. In the first module, traffic rules are encoded into a machine computable (MC) format. In the second module, AV information is formulated into the MC format to comply with the encoded traffic rules. Finally, in module three the mapping and reasoning of traffic rules and AV information are combined to assess the AV behaviour. A brief description of each module is given below.

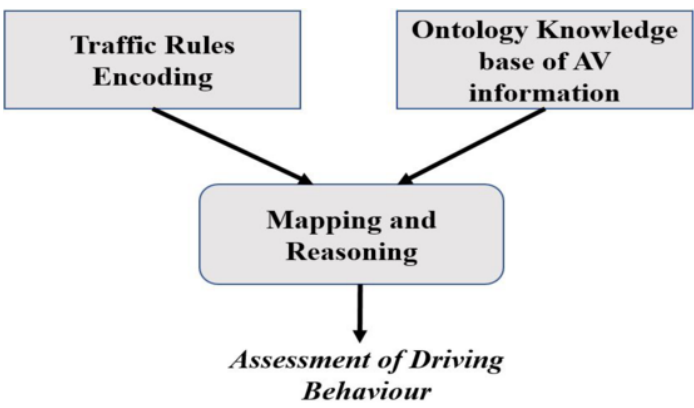

Figure 1. Flow diagram of driving behaviour assessment methodology.

\subsection{Traffic Rules Encoding}

Defeasible Deontic Logic (DDL) is used as a formal foundation of this encoding methodology [13]. The proposed methodology works in four steps, as shown in Figure 2, which are define atoms, identify norms, generate if-then structure, and rules encoding.

In the first step, atoms are defined based on the terms appearing in the traffic rules. An atom corresponds to a statement (combining terms in the traffic rules) that can be evaluated as true or false. A term is a variable or an individual constant in the sentence. The proposed encoding method considers these variables and constants in the rule sentences. Norms are identified in the second step. In the traffic rule, norms are conditions to perform specific actions. Every norm is represented by one or more rules, which could either be constitutive or prescriptive rules. Both constitutive and prescriptive forms of rules are used to identify norms. In the third step, if-then structures are generated from rules using atoms and norms. This structure comprises two parts: if (antecedent or premise) and then (consequent or conclusion). If the premise becomes true, then the consequent 


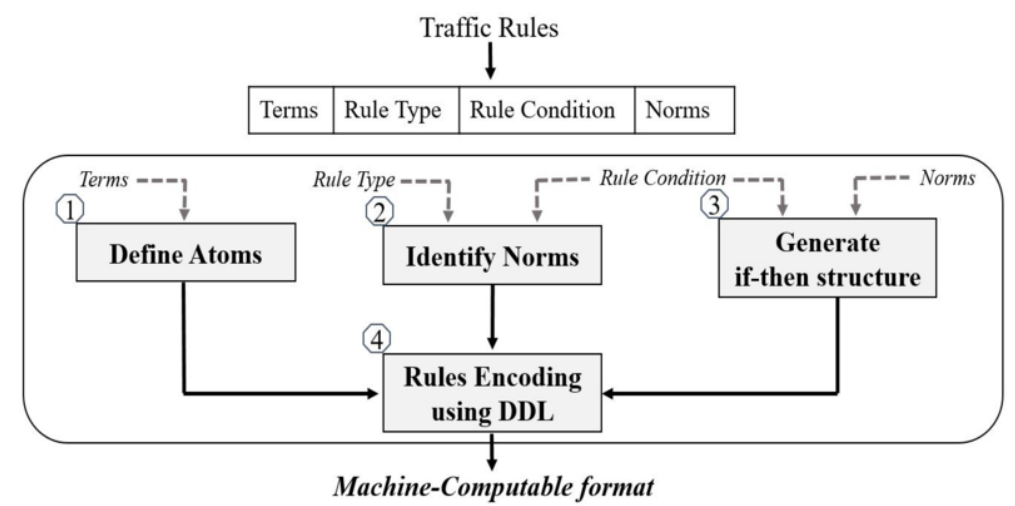

Figure 2. Traffic rules encoding.

part of the rules is triggered. In the fourth step, rules are encoded into the MC format. After identifying and combining atoms, norms, and if-then structures, DDL is applied to them to create the MC format of the rule. The normative effects of (prescriptive) rules are modelled by Obligation $(O)$, Prohibition $(F)$, and Permission $(P)$.

We now provide (Figure 3 ) an example of traffic rules encoding using DDL. For this example, we use Queensland Overtaking Traffic Rules $141^{4}$. In the bottom of Figure 3, the priority between the encoded rule is shown.

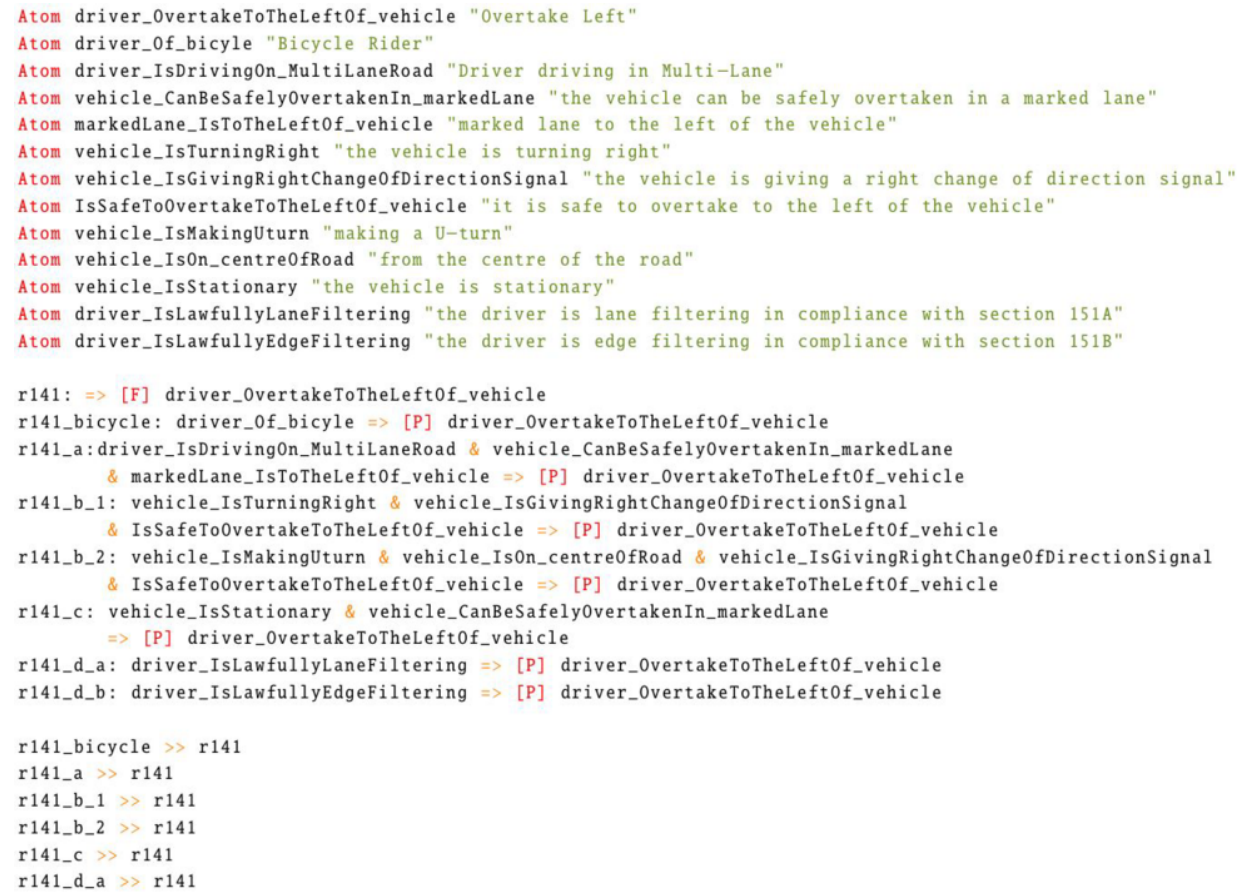

Figure 3. Encoding of Queensland Overtaking Trafic Rule 141 


\subsection{Ontology Knowledge Base}

Ontology is a way of representing knowledge in a structured framework that consists of concepts (classes) and relationships (properties). It allows communication and information sharing between software and hardware agents by facilitating the design of rigorous and exhaustive conceptual schema. An important characteristic of ontology is that it represents knowledge in a machine-computable (MC) format as RDF (Resource Description Framework) data [14]. RDF ${ }^{5}$ provides a conceptual statement to give a clear specification for modelling data. This MC knowledge (RDF) representation can bridge the gap between AV perception and knowledge processing. Therefore, in this work, we create ontologies of AV information. Moreover, it is also proved by [15] that an ontology can effectively represent road information and driving behaviour of the vehicle, which is helpful for AV knowledge processing. Here, the MC knowledge base is used by the encoded traffic rules to provide the input for the reasoning engine about what are the legal requirements for the AV in the particular situation identified by the data available to the AV.

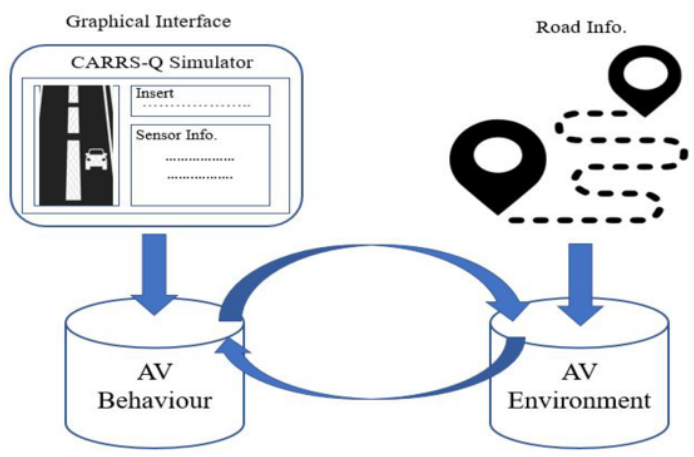

Figure 4. Structure of Knowledge Base.

The structure of the knowledge base is shown in Figure 4. Protégé6 is used to build these ontologies. The knowledge base consists of two ontologies: AV behaviour and AV environment ontology. AV behaviour ontology is created by using the behaviour information (i.e speed, direction, lane number, etc.) of the AV. The environment ontology is created by using road information (i.e road marking, road type, etc.) and information about AV surroundings (i.e other vehicles speeds, other vehicles lane numbers, etc.). We collect all this information from the CARRS-Q advanced driving simulator ${ }^{7}$. Moreover, based on the requirements, these ontologies can be reused and easily extended by adding another concept. To design the road in the simulator, we collect road information (Queensland, Australia) from Wikipedia and other web blogs 8 .

\subsection{Reasoning}

This section will introduce the reasoning engine to make the assessment of the AV driving behaviour against traffic rules. Figure 5 shows the work flow diagram of the reasoning

${ }^{5}$ https://www.w3.org/RDF/

${ }^{6}$ https://protege.stanford.edu/

${ }^{7} \mathrm{https} / / /$ research.qut.edu.au/carrsq/services/advanced-driving-simulator/

${ }^{8}$ https://www.ozroads.com.au/QLD/classifications.htm 
engine. The input to this reasoning engine are atoms (from encoded traffic rules), encoded traffic rules, and knowledge base. The proposed reasoning engine works in four steps. Brief descriptions of these four steps are given below.

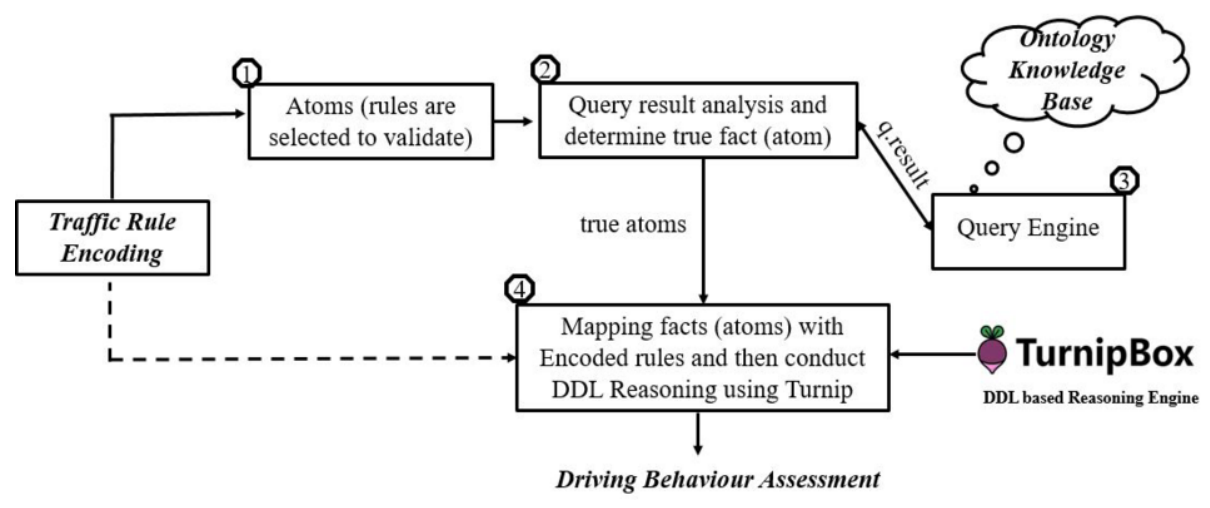

Figure 5. Work flow diagram of the Reasoning Engine.

\subsubsection{Atoms:}

The generated atoms of corresponding traffic rules are stored in this step for further processing.

\subsubsection{Determine True Fact}

This step determines true facts (atoms) for the driving action of the AV. In this step, for each query, we set some predefined answers. The query result is compared with those answers and if it matches then the system identifies that it is a true fact. For example, to verify the atom (driver_Of_bicyle), the SPARQL Query 1_1 is triggered. The answer of the query shows that it is AV \& Automated_Vehicle. Therefore, it can be concluded that, this atom is not true as the atom is about a bicycle.

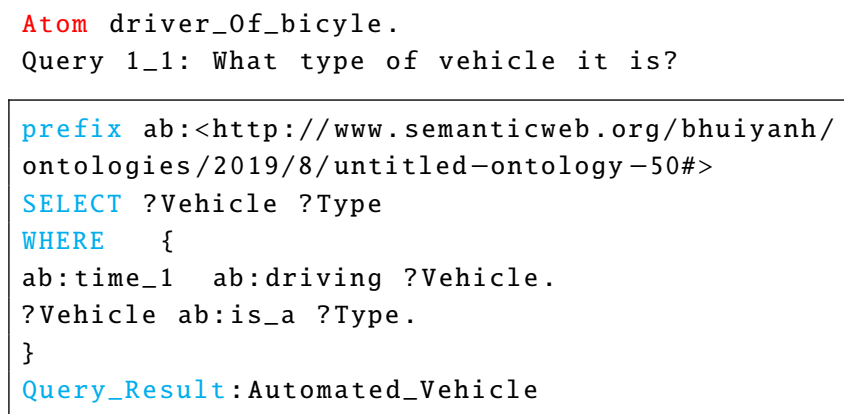

\subsubsection{Query Engine}

The query engine contains predefined SPARQL queries for each atom. These queries are made based on the empirical study of the overtaking traffic rules of Queensland. Based on the atom, the number of queries vary. SPARQL is one of the most powerful and effective query languages to access the ontology-based knowledge base. Here, we use SPARQL 
queries to retrieve AV behaviour and environment information from the knowledge base. An algorithm is designed to trigger these queries. If the query result is NULL, then the process breaks and uses the next query. An example of an atom (driver_Of_bicyle) and its corresponding query and its results is shown above.

\subsubsection{Mapping and Reasoning in Turnip}

Turnip ${ }^{9}$ is a Defeasible Deontic Logic-based reasoning tool. It is a tool which accepts facts (atoms), strict rules, defeasible rules, defeaters, superiority relation, and modality of DL. It supports non-monotonic and monotonic reasoning with incomplete and inconsistent information. A full illustration of Turnip is out of the scope of this paper. In this research, Turnip receives the encoded rules and atoms and thus does the mapping and reasoning.

For example (see Table 1), regarding overtaking traffic rule 141 (Figure 3), if for any timestamp, true facts for the AV are as Table 1(a), then the reasoning result shows that, $\mathrm{AV}$ has permission $([P])$ to do left-side overtaking. However, if any of the facts among them (Table 1(a)) become false like (Table 1(b)), then permisssion for left overtaking is declined $([F])$ according to traffic rule 141 .

Table 1. Example of mapping and reasoning in Turnip

\begin{tabular}{|c|c|}
\hline \multicolumn{2}{|c|}{ Rules } \\
\hline \multicolumn{2}{|c|}{ Encoding of Rule 141 (Figure 3) } \\
\hline True Facts & True Facts \\
\hline $\begin{array}{l}\text { driver_IsDrivingOn_MultiLaneRoad } \\
\text { vehicle_CanBeSafelyOvertakenIn_markedLane } \\
\text { markedLane_IsToTheLeftOf_vehicle } \\
\text { IsSafeToOvertakeToTheLeftOf_vehicle } \\
\text { vehicle_IsOn_centreOfRoad }\end{array}$ & $\begin{array}{l}\text { driver_IsDrivingOn_MultiLaneRoad } \\
\text { vehicle_CanBeSafelyOvertakenIn_markedLane } \\
\text { IsSafeToOvertakeToTheLeftOf_vehicle } \\
\text { vehicle_IsOn_centreOfRoad }\end{array}$ \\
\hline Results & Results \\
\hline $\begin{array}{l}\text { [P] driver_overtakeToThe } \\
\text { Leftof_vehicle }\end{array}$ & $\begin{array}{l}\text { [F] driver_overtakeToThe } \\
\text { Leftof_vehicle }\end{array}$ \\
\hline (a) & (b) \\
\hline
\end{tabular}

\section{Experiment}

This chapter shows the experiment results of the proposed Automated Vehicle (AV) driving behaviour assessment approach. We firstly present the experiment scenarios and data. Each scenario is a specific maneuver of the AV. The experiment is conducted to find the legal and illegal driving behaviour of the AV during the maneuver. The evaluation is performed with the help of domain experts.

\subsection{Experiment Scenarios}

The CARRS-Q advanced driving simulator is used to make experiment scenarios. We do some empirical study on overtaking cases of Queensland traffic and hence composed scenarios. This study helps us to cover (see Figure 6) almost all aspects of overtaking cases generally occurring in Queensland. Four scenarios are designed to investigate the proposed approach. A depiction of each scenario is shown in Figure 6.

\footnotetext{
${ }^{9}$ https://turnipbox.netlify.com/
} 
- In Figure 6(a), the AV is approaching to overtake the TV-1 in a multi-lane road.

- AV is approaching to overtake TV-2 although it is displaying a "do not overtake turning vehicle" sign (Figure 6(b)).

- In Figure 6(c), the AV is approaching to overtake TV-1 as it is in a stationary position.

- In a non-marked two-way road, the AV is approaching to overtake TV-1 (Figure $6(d))$.
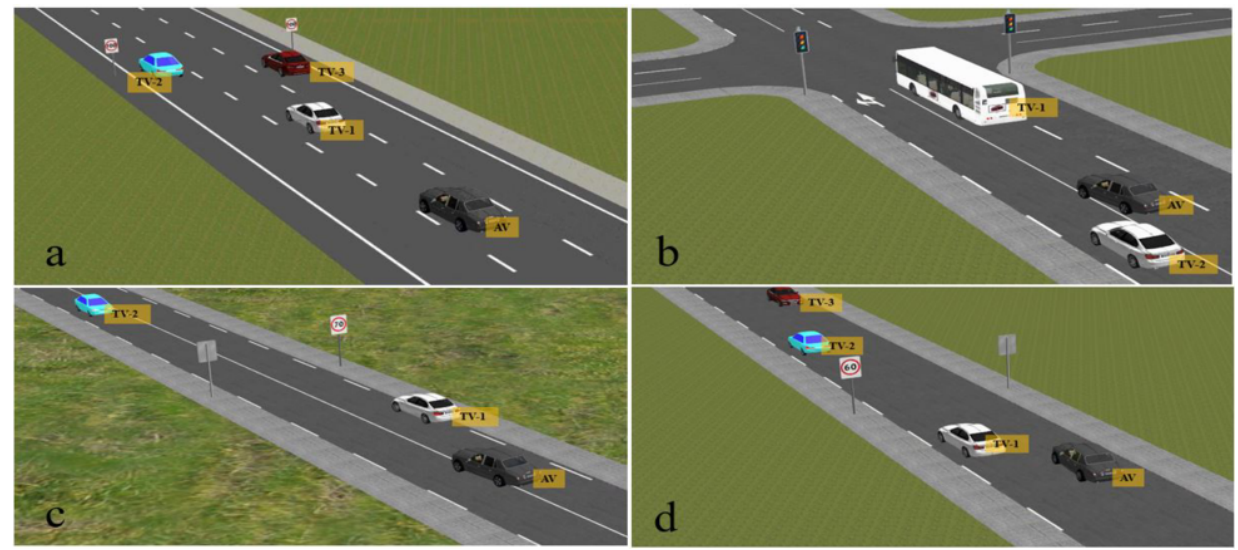

Figure 6. Experiment Scenarios.

These types of overtaking cases are very common in Queensland traffic. In some aspects, these types of maneuver are risky and challenging. We experiment on these four scenarios for both Left Overtaking (LO) and Right Overtaking (RO). Based on overtaking type (LO / RO), the scenario changes. For each experiment, we consider three different maneuvers to evaluate the proposed methodology effectiveness. Among these maneuvers, two of them are a clear case of legal and illegal action. The third maneuver is about the border-line maneuver, which cannot directly define whether it is legal or illegal.

\subsection{Experiment Data}

Experiment data is generated using the CARRS-Q simulator. The simulator can provide the data under managed and repeatable conditions and also make the data more useful and meaningful for analysing. A snippet of experiment data is shown in Figure 7. Here, we generate behaviour and environment information of vehicles every $0.05 \mathrm{~s}$.

\subsection{Experiment Result}

We conducted 24 experiments based on the above-represented scenarios (Figure 6). 12 experiments were conducted individually for Left Overtaking (LO) and Right Overtaking (RO). Each experiment is divided into n timestamps. Each timestamp is 0.05s (Figure 7). In each experiment, every timestamp is validated against the corresponding traffic rule. After completing the validation of all timestamps of an experiment, the result is determined. For example, experiment result of all timestamps of the LO, experiment 2, maneuver type -3 is shown in Figure 7. As in this maneuver, in some timestamps the driving action is prohibited (Prohibition: $F$ ), therefore this maneuver is illegal according 


\begin{tabular}{|c|c|c|c|c|c|c|c|c|c|c|c|c|c|c|c|c|}
\hline Timestamp & $\underset{\text { speed }}{\mathrm{AV}}$ & $\begin{array}{c}\mathrm{AV} \\
\text { acceleration }\end{array}$ & $\cdot$ & - & $\underset{\text { positionx }}{\text { AV }}$ & $\begin{array}{c}\mathrm{AV} \\
\text { positiony }\end{array}$ & $\underset{\text { lanenumber }}{\mathrm{AV}}$ & $\begin{array}{l}\text { TV-1 } \\
\text { speed }\end{array}$ & $\begin{array}{c}\text { TV-1 } \\
\text { acceleration }\end{array}$ & $\cdot$ & - & $\begin{array}{c}\text { TV-1 } \\
\text { positionx }\end{array}$ & $\begin{array}{c}\text { TV-1 } \\
\text { positiony }\end{array}$ & $\cdot$ & . & Assessment \\
\hline 0.15 & 1.76 & 2.70 & - & - & 208.36 & 121.65 & 2 & 10.02 & 0.12 & - & . & 198.63 & 120.19 & - & . & $\mathbf{P}$ \\
\hline - & - & - & - & - & - & - & - & - & . & . & . & . & - & - & . & . \\
\hline 2.9 & 29.56 & 5.19 & - & - & 214.98 & 125.39 & 2 & 23.49 & 1.48 & - & - & 209.50 & 126.34 & - & - & $\mathbf{P}$ \\
\hline 2.95 & 30.46 & 5.20 & - & - & 215.33 & 125.5941 & 2 & 23.75 & 1.48 & - & - & 209.78 & 126.50 & - & . & $\mathbf{P}$ \\
\hline . & - & - & . & - & . & . & . & . & . & - & - & . & . & . & - & . \\
\hline 5.15 & 62.49 & 3.23 & - & - & 245.70 & 143.4 & 2 & 29.28 & -0.41 & . & - & 246.31 & 147.08 & . & - & F \\
\hline 5.2 & 63.05 & 3.23 & - & - & 246.41 & 143.86 & 2 & 29.20 & -0.45 & - & - & 246.67 & 147.28 & - & - & F \\
\hline - & - & - & - & - & - & - & - & - & - & - & - & - & - & - & - & . \\
\hline 7.35 & 88.25 & 2.44 & - & - & 280.43 & 166.31 & 1 & 34.70 & -1.10 & . & . & 245.19 & 146.45 & . & . & $\mathbf{P}$ \\
\hline
\end{tabular}

Figure 7. An snippet of experiment data and assessment result (LO, Ex -2, Maneuver Type-3).

to the LO-141 (QLD Traffic Rules). However, if all timestamps of this maneuver are permitted (Permission: $P$ ), then it would become a legal maneuver.

Table 2 shows the effectiveness of the proposed methodology in terms of assessing AV behaviour against overtaking traffic rules. To evaluate the experiment result, we took help from three domain experts (who have 25 years experience of driving in Queensland and never have any allegation of illegal overtaking). We use the knowledge of experienced drivers to validate the interpretation of local overtaking maneuvers. For the maneuver, we consider domain expert judgement as the ground truth. If the experts regard any behaviour as illegal then the result is considered negative.

According to the experiment result (Table 2), the proposed methodology successfully works for both $\mathrm{LO}$ and $\mathrm{RO}$ cases for the experiment 2 . For experiment- $3 \& 4$, the proposed method could correctly assess all LO cases, but is unsuccessful for all RO cases. On the

Table 2. Experiment Result of the proposed assessment method.

\begin{tabular}{|c|c|c|c|c|c|c|c|}
\hline \multirow{3}{*}{ Ex-No. } & \multirow{3}{*}{$\begin{array}{c}\text { Situations } \\
\text { Covered }\end{array}$} & \multicolumn{6}{|c|}{ Overtaking Type } \\
\hline & & \multicolumn{3}{|c|}{ Left Overtaking (LO) } & \multicolumn{3}{|c|}{ Right Overtaking (RO) } \\
\hline & & $\begin{array}{c}\text { Maneuver } \\
\text { Type }\end{array}$ & $\begin{array}{c}\text { Proposed } \\
\text { Methodology }\end{array}$ & $\begin{array}{c}\text { Domain } \\
\text { Expert }\end{array}$ & $\begin{array}{c}\text { Maneuver } \\
\text { Type }\end{array}$ & $\begin{array}{c}\text { Proposed } \\
\text { Methodology }\end{array}$ & $\begin{array}{l}\text { Domain } \\
\text { Expert }\end{array}$ \\
\hline \multirow{3}{*}{ Ex-1 } & \multirow{3}{*}{$\begin{array}{l}\text { Vehicles position, } \\
\text { multiple vehicles, multiple } \\
\text { lanes, lane type (marked } \\
\text { lane), lane marking. }\end{array}$} & Type - 1 & $\checkmark$ & $\checkmark$ & Type - 1 & $\checkmark$ & $\checkmark$ \\
\hline & & Type -2 & $x$ & $x$ & Type - 2 & $x$ & $x$ \\
\hline & & Type -3 & $\times$ & $\checkmark$ & Type -3 & $x$ & $x$ \\
\hline \multirow{3}{*}{ Ex-2. } & \multirow{3}{*}{$\begin{array}{l}\text { Vehicles position, multiple } \\
\text { vehicles, multiple lanes, } \\
\text { lane type (marked } \\
\text { lane), lane marking, } \\
\text { do not overtake turning } \\
\text { vehicle sign, Intersections. }\end{array}$} & Type -1 & $\checkmark$ & $\checkmark$ & Type -1 & $\checkmark$ & $\checkmark$ \\
\hline & & Type - 2 & $x$ & $x$ & Type - 2 & $x$ & $x$ \\
\hline & & Type -3 & $x$ & $x$ & Type -3 & $x$ & $x$ \\
\hline \multirow[b]{3}{*}{ Ex-3 } & \multirow{3}{*}{$\begin{array}{l}\text { Vehicles position } \\
\text { multiple vehicles, stationary } \\
\text { vehicle, two-way lane, } \\
\text { lane type (marked } \\
\text { lane), lane marking. }\end{array}$} & Type -1 & $\checkmark$ & $\checkmark$ & Type -1 & $\checkmark$ & $\checkmark$ \\
\hline & & Type -2 & $x$ & $\times$ & Type - 2 & $\times$ & $x$ \\
\hline & & Type -3 & $\times$ & $\checkmark$ & Type -3 & $x$ & $\checkmark$ \\
\hline \multirow[b]{3}{*}{ Ex-4. } & \multirow{3}{*}{$\begin{array}{l}\text { Vehicles position } \\
\text { multiple vehicles, multiple } \\
\text { lanes, lane type } \\
\text { (non-marked lane), } \\
\text { two-way lane. }\end{array}$} & Type -1 & $\checkmark$ & $\checkmark$ & Type -1 & $\checkmark$ & $\checkmark$ \\
\hline & & Type - 2 & $\times$ & $x$ & Type -2 & $x$ & $x$ \\
\hline & & Type -3 & $\times$ & $x$ & Type -3 & $\checkmark$ & $\checkmark$ \\
\hline
\end{tabular}


other side, for experiment-1, the proposed method is not successful to correctly assess all LO cases, while it is successful for all RO cases.

\section{Conclusion and Future Work}

The experiment result shows that the proposed assessment method can assess the AV driving behaviour against traffic rules by effectively handling exceptions and resolving conflicts in rule norms. Therefore, it can be said that, this assessment methodology would be useful for the traffic authority to automatically identify AVs that drive illegally.

In future, we will enhance the scope of this proposed assessment mechanism by covering other traffic environments such as lane change, roundabout, intersection crossing, and etc. Furthermore, from this assessment mechanism we will determine which traffic rules need additional interpretation in terms of the information available by an AV.

\section{References}

[1] Leenes R, Lucivero F. Laws on robots, laws by robots, laws in robots: regulating robot behaviour by design. Law, Innovation and Technology. 2014 Dec 31;6(2):193-220.

[2] Khorasani G, Tatari A, Yadollahi A, Rahimi M. Evaluation of intelligent transport system in road safety. International Journal of Chemical, Environmental \& Biological Sciences (IJCEBS). 2013;1(1):110-8.

[3] Fulbright NR. Autonomous vehicles: The legal landscape of dedicated short range communication in the US, UK and Germany. Accessed: Dec. 2017;11:2018.

[4] Rizaldi A, Keinholz J, Huber M, Feldle J, Immler F, Althoff M, Hilgendorf E, Nipkow T. Formalising and monitoring traffic rules for autonomous vehicles in Isabelle/HOL. In International Conference on Integrated Formal Methods 2017 Sep 20 (pp. 50-66). Springer, Cham.

[5] Prakken H. On the problem of making autonomous vehicles conform to traffic law. Artificial Intelligence and Law. 2017 Sep 1;25(3):341-63.

[6] Governatori G. The Regorous approach to process compliance. In2015 IEEE 19th International Enterprise Distributed Object Computing Workshop 2015 Sep 21 (pp. 33-40). IEEE.

[7] Zhao L, Ichise R, Liu Z, Mita S, Sasaki Y. Ontology-based driving decision making: A feasibility study at uncontrolled intersections. IEICE TRANSACTIONS on Information and Systems. 2017 Jul $1 ; 100(7): 1425-39$.

[8] Xiong Z, Dixit VV, Waller ST. The development of an Ontology for driving Context Modelling and reasoning. In 2016 IEEE 19th International Conference on Intelligent Transportation Systems (ITSC) 2016 Nov 1 (pp. 13-18). IEEE.

[9] Buechel M, Hinz G, Ruehl F, Schroth H, Gyoeri C, Knoll A. Ontology-based traffic scene modeling, traffic regulations dependent situational awareness and decision-making for automated vehicles. In 2017 IEEE Intelligent Vehicles Symposium (IV) 2017 Jun 11 (pp. 1471-1476). IEEE.

[10] Shadrin SS, Varlamov OO, Ivanov AM. Experimental autonomous road vehicle with logical artificial intelligence. Journal of advanced transportation. 2017 Jan 1;2017.

[11] Costescu DM. Keeping the autonomous vehicles accountable: Legal and Logic Analysis on Traffic Code. In Conference Vision Zero for Sustainable Road Safety in Baltic Sea Region 2018 Dec 5 (pp. 21-33). Springer, Cham.

[12] Aladin DV, Varlamov OO, Chuvikov DA, Chernenkiy VM, Smelkova EA, Baldin AV. Logic-based artificial intelligence in systems for monitoring the enforcing traffic regulations. InIOP Conference Series: Materials Science and Engineering 2019 May (Vol. 534, No. 1, p. 012025). IOP Publishing.

[13] Bhuiyan H, Olivieri F, Governatori G, Badiul M Islam, Bond A, Rakotonirainy A. A Methodology for Encoding Regulatory Rules. In 2019 4th International Workshop on MIning and REasoning on Legal texts (MIREL) 2019 Dec 11 (pp. 1-13). CUER-WS.

[14] Najmi E, Malik Z, Hashmi K, Rezgui A. ConceptRDF: An RDF presentation of ConceptNet knowledge base. In 2016 7th International Conference on Information and Communication Systems (ICICS) 2016 Apr 5 (pp. 145-150). IEEE.

[15] Zhao L, Ichise R, Mita S, Sasaki Y. Core Ontologies for Safe Autonomous Driving. InInternational Semantic Web Conference (Posters \& Demos) 2015. 